\title{
Mycobacterium microti Infection in Free-Ranging Wild Boar, Spain, 2017-2019
}

\section{Bernat Pérez de Val, Albert Sanz, Mercè Soler, Alberto Allepuz, Lorraine Michelet, María Laura Boschiroli, Enric Vidal}

Author affiliations: Institut de Recerca i Tecnologia AgroalimentàriesCentre de Recerca en Sanitat Animal, Bellaterra, Spain (B. Pérez de Val, A. Allepuz, E. Vidal); Departament d'Agricultura, Ramaderia, Pesca i Alimentació de la Generalitat de Catalunya, Barcelona, Spain (A. Sanz, M. Soler); Universitat Autònoma de Barcelona, Bellaterra (A. Allepuz); Agence Nationale de Sécurité Sanitaire de l'Alimentation, de l'Environnement et du Travail, Maisons-Alfort, France (L. Michelet, M.L. Boschiroli)

DOI: https://doi.org/10.3201/eid2511.190746

Mycobacterium microti is a member of the Mycobacterium tuberculosis complex that causes pathology in many mammals. M. microti infections have been found in some countries in Europe. We report an outbreak of tuberculosis caused by M. microti in wild boars in Spain.

$M$ cobacterium microti is a member of the Mycobacterium tuberculosis complex (MTBC), which also includes M. tuberculosis and M. bovis, the main causes of human and animal tuberculosis (TB), respectively. Even though voles and other wild small rodents were initially identified as its natural hosts (1), M. microti can cause pathology in a wide range of mammals, including pets, livestock, wildlife (2-5), and humans (6). M. microti infections have been previously reported in several countries in Europe, including Switzerland, Italy, and France $(3,7-9)$. We report an outbreak of tuberculosis caused by $M$. microti in free-ranging wild boars in the Iberian Peninsula in Spain.

During June 2017-March 2019, a total of 9 free-ranging wild boars with lesions associated with TB were detected in the outbreak area, covering $\approx 3,000$ hectares in the Catalan Pyrenees (Figure). TB was confirmed histologically, first by hematoxylin and eosin staining (9/9) and then by Ziehl-Neelsen staining of acid-fast bacilli (7/9). In all cases, submandibular lymph nodes showed granulomatous necrotizing lymphadenitis, sometimes with scant acid-fast bacilli, similar to that found in M. microti infections previously described in wild boar, which were generally confined to lymph nodes in the head $(7,8)$.

To confirm the causative agent for these infections, we extracted DNA from tissue samples (DNAExtract-VK, Vacunek, http://vacunek.com) and performed real-time PCR (TBC-VK, Vacunek), which confirmed MTBC in 6 of 9 suspected cases. DVR spoligotyping at VISAVET Health Surveillance Centre, Universidad Complutense de Madrid, identified the pathogen in 4 of the 6 confirmed MTBC cases as M. microti (spoligopattern SB0423; Mycobacterium bovis spoligotype database, http://www.mbovis.org). In the other
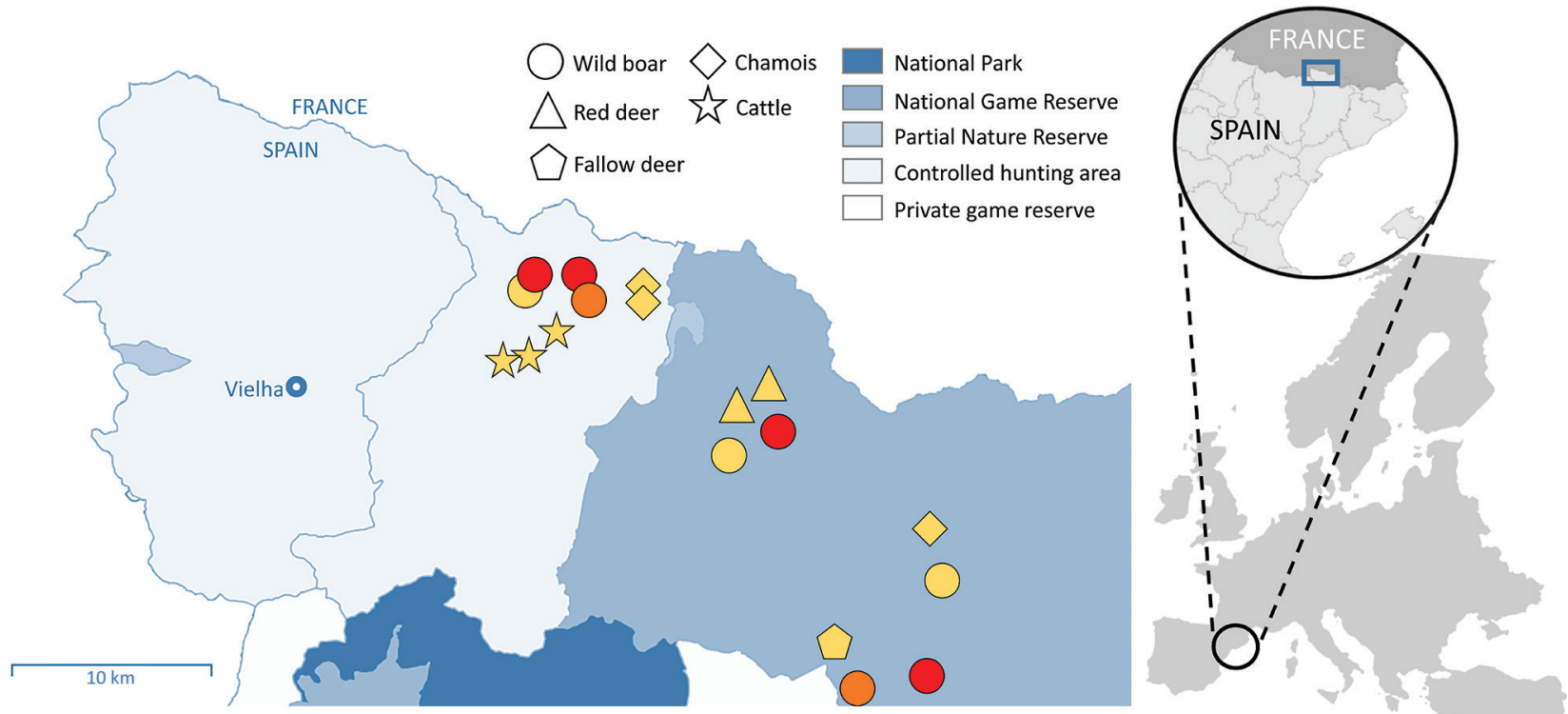

Figure. Outbreak area for wild boar tuberculosis (TB) cases, Spain, June 2017-March 2019. Circles show cases of wild boars with TB lesions. Stars indicate the location of cattle herds with positive skin tests (not confirmed at slaughterhouse); triangles, pentagons, and diamonds show locations of Mycobacterium tuberculosis complex-seropositive ungulates (no tissue samples were obtained from these animals). Colors indicate etiologic agent identification: red, $M$. microti; orange, any $M$. tuberculosis complex; yellow, unidentified. Different hunting areas are indicated. The border between Spain and France and the main village (Vielha) are labelled. Inset maps show location of the study area on the Iberian Peninsula. 
2 cases, the laboratory was unable to determine the species because of low DNA load from the sample. This result likely was due to the slow in vitro growth rate of $M$. microti in infected animals, which makes it difficult to isolate in routine diagnostic laboratories.

Spoligopattern SB0423 is included in a phylogenetic cluster with spoligopattern SB0112, also associated with $M$. microti, on the basis of neighbor joining. Both spoligotypes are localized in the eastern French Pyrenees, close to the borders with Spain and Andorra (3). Most M. microti cases in France, found in cats, dogs, and llamas during 2005-2016 and, since 2015, in wild boars and badgers, have been found within $50 \mathrm{~km}$ of the outbreak area in Spain. The 2017 M. microti cases described in this report were found closer to the border with France; the remaining 2 cases, detected in 2019, were localized near the southern limit of the outbreak area (Figure).

In the outbreak area, up to 18 animals in 3 cattle herds showed positive results for a single intradermal tuberculin skin test (Figure). However, none of these animals showed gross lesions in target tissues (i.e., lungs, pulmonary, and retropharyngeal lymph nodes) at a slaughterhouse or positive results to mycobacterial culture and PCR. Similarly, in a recent case in France, a cow reacting to a TB skin test did not show TB-like lesions in respiratory tissues and returned negative results from cultures, but $M$. microti DNA was finally detected in retropharyngeal lymph nodes only by using advanced molecular techniques (5). These results indicate that cattle exposed to M. microti may induce positive results to diagnostic tests performed in bovine TB eradication campaigns. $M$. microti infection can sometimes cause visible lesions in cattle (2), but the fact that M. microti are natural knockouts for the virulence-related RD1 ${ }^{\text {mic }}$ genomic region (10) may indicate a lower pathogenicity compared with other MTBC species and account for these negative test results.

We tested additional wild ungulates in the outbreak area and found that 6 ( 2 red deer, 1 fallow deer, and 3 chamois) were seropositive for MTBC using a MPB83-specific IgG indirect ELISA test (Figure). Unfortunately, no tissue samples were submitted to examine for lesions or to detect and identify mycobacteria. However, overall positive results for M. microti and the absence of other MTBC strains during the 2017-2019 period in the outbreak area suggest a multihost circulation of $M$. microti. Because voles are known maintenance hosts of $M$. microti (1), further investigation of wild small rodent populations in the outbreak area could determine the epidemiology of this outbreak in greater detail.

These findings, together with previously reported cases nearer the border between France and Spain, indicate a transboundary circulation of $M$. microti across the Pyrenean border that should be taken into account for wildlife
TB surveillance. Coordinated action between animal health authorities and laboratories in Spain and France is required, as well as the improvement of livestock management and biosecurity practices.

\section{Acknowledgments}

We thank the forest guard body of the Catalan Government and Vedat Pirineus S.L. for wildlife sample collection. We are also grateful to Maite Martín, Zoraida Cervera, Sierra Espinar, and Mónica Pérez for their outstanding technical assistance.

This work was funded by the Department of Agriculture, Livestock, Fisheries and Food (DARP) of the Government of Catalonia. IRTA is supported by Centres de Recerca de Catalunya (CERCA) Programme/Generalitat de Catalunya (www.cerca.cat).

\section{About the Author}

Dr. Pérez de Val is a researcher at Institut de Recerca $\mathrm{i}$ Tecnologia Agroalimentàries-Centre de Recerca en Sanitat Animal, Bellaterra, Spain, where he conducts research on animal TB.

\section{References}

1. Cavanagh R, Begon M, Bennett M, Ergon T, Graham IM, De Haas PE, et al. Mycobacterium microti infection (vole tuberculosis) in wild rodent populations. J Clin Microbiol. 2002;40:3281-5. https://doi.org/10.1128/JCM.40.9.3281-3285.2002

2. Smith NH, Crawshaw T, Parry J, Birtles RJ. Mycobacterium microti: more diverse than previously thought. J Clin Microbiol. 2009;47:2551-9. https://doi.org/10.1128/JCM.00638-09

3. Michelet L, de Cruz K, Karoui C, Hénault S, Boschiroli M. Mycobacterium microti, an unrecognized tubercular agent [in French]. Epidémiol Santé Anim. 2017;71:129-38.

4. Michelet L, de Cruz K, Phalente Y, Karoui C, Hénault S, Beral M, et al. Mycobacterium microti infection in dairy goats, France. Emerg Infect Dis. 2016;22:569-70. https://10.3201/ eid2203.151870

5. Michelet L, De Cruz K. Mycobacterium microti infection in a cow in France. Vet Rec. 2017;180:429. PubMed http://dx.doi. org/10.1136/vr.j2041

6. Panteix G, Gutierrez MC, Boschiroli ML, Rouviere M, Plaidy A, Pressac D, et al. Pulmonary tuberculosis due to Mycobacterium microti: a study of six recent cases in France. J Med Microbiol. 2010;59:984-9. https://doi.org/10.1099/ jmm.0.019372-0

7. Schöning JM, Cerny N, Prohaska S, Wittenbrink MM, Smith NH, Bloemberg G, et al. Surveillance of bovine tuberculosis and risk estimation of a future reservoir formation in wildlife in Switzerland and Liechtenstein. PLoS One. 2013;8:e54253. https://doi.org/10.1371/journal.pone.0054253

8. Boniotti MB, Gaffuri A, Gelmetti D, Tagliabue S, Chiari M, Mangeli A, et al. Detection and molecular characterization of Mycobacterium microti isolates in wild boar from northern Italy. J Clin Microbiol. 2014;52:2834-43. https://doi.org/10.1128/ JCM.00440-14

9. Chiari M, Ferrari N, Giardiello D, Avisani D, Pacciarini ML, Alborali L, et al. Spatiotemporal and ecological patterns of 
Mycobacterium microti infection in wild boar (Sus scrofa). Transbound Emerg Dis. 2016;63:e381-8. https://doi.org/10.1111/ tbed.12313

10. Brodin P, Eiglmeier K, Marmiesse M, Billault A, Garnier T, Niemann $\mathrm{S}$, et al. Bacterial artificial chromosome-based comparative genomic analysis identifies Mycobacterium microti as a natural ESAT-6 deletion mutant. Infect Immun. 2002;70:5568-78. https://doi.org/10.1128/IAI.70.10.5568-5578.2002

Address for correspondence: Bernat Pérez de Val, Institut de Recerca i Tecnologia Agroalimentàries-Centre de Recerca en Sanitat Animal, Edifici CReSA. Campus UAB (Bellaterra), Bellaterra, Barcelona 08193, Spain; email: bernat.perez@irta.cat

\section{Availability of Injectable Antimicrobial Drugs for Gonorrhea and Syphilis, United States, 2016}

\section{William S. Pearson, Donald K. Cherry, Jami S. Leichliter, Laura H. Bachmann, Nicole A. Cummings, Matthew Hogben}

Author affiliations: Centers for Disease Control and Prevention, Atlanta, Georgia, USA (W.S. Pearson, J.S. Leichliter, L.H. Bachmann, M. Hogben); Centers for Disease Control and Prevention, Hyattsville, Maryland, USA (D.K. Cherry, N.A. Cummings)

DOI: https://doi.org/10.3201/eid2511.190764

We estimated the availability of the injectable antimicrobial drugs recommended for point-of-care treatment of gonorrhea and syphilis among US physicians who evaluated patients with sexually transmitted infections in 2016. Most physicians did not have these drugs available on-site. Further research is needed to determine the reasons for the unavailability of these drugs.

$\mathrm{R}$ ates of sexually transmitted infections (STIs) are on the rise in the United States. Compared with the incidence in 2013, the numbers of reported gonorrhea $(+75 \%)$ and syphilis (primary and secondary, $+153 \%$ ) cases in 2017 were dramatically increased (1). Timely (optimally sameday) treatment of bacterial STIs with a highly effective regimen is critical for national STI control efforts and can help mitigate the development of drug resistance, a particularly pertinent issue for Neisseria gonorrhoeae (2). In addition, the high number of congenital syphilis cases in the United States in 2017 highlights the need for efficient and effective treatment of Treponema pallidum infection (3).

The recommended first-line treatment for uncomplicated gonorrhea is intramuscular ceftriaxone $(250 \mathrm{mg})$ and for primary and secondary syphilis is intramuscular penicillin $\mathrm{G}$ benzathine (2.5 million units) (4). On-site access to these injectable medications in clinics facilitates point-of-care treatment and helps mitigate drug resistance to $N$. gonorrhoeae and T. pallidum when these drugs are used instead of the oral antimicrobial drug alternatives. Therefore, we set out to determine a nationally representative estimate of the availability of these medications and examine if differences existed by geographic location and between offices designated and not designated as patient-centered medical homes (PCMHs).

We used the 2016 Physician Induction File of the National Ambulatory Medical Care Survey (5) to assess the number of physicians who treat patients with STIs and had injectable antimicrobial drugs available on-site. The Physician Induction File is a nationally representative survey of office-based, nonfederal physicians in the United States and includes information regarding practice characteristics and habits. Use of the data is restricted, and access is facilitated through the Research Data Center, National Center for Health Statistics, Centers for Disease Control and Prevention, in Hyattsville, Maryland, USA. A total of 1,030 physicians (46.2\% unweighted response rate), representing an estimated 330,581 $(95 \%$ CI 326,994-334,168) physicians in the United States, completed the Physician Induction File in 2016. In this survey, physicians who reported evaluating or treating patients for STIs were asked which antimicrobial drugs they had available on-site for sameday management of gonorrhea and syphilis, including intramuscular ceftriaxone and penicillin $\mathrm{G}$ benzathine at the recommended doses. We determined national estimates of reported on-site, same-day availability for these antimicrobial drugs and stratified results by PCMH designation and US region. We used multiple logistic regression models to determine if $\mathrm{PCMH}$ designation and region were predictive of on-site availability of these 2 medications.

An estimated $45.2 \%(149,483,95 \%$ CI 138,850 $160,116)$ of office-based physicians indicated that they evaluate patients for STIs in their offices. Of these, $77.9 \%$ $(116,479,95 \%$ CI 105,360-127,598) reported not having penicillin $\mathrm{G}$ benzathine available on-site and $56.1 \%$ $(83,827,95 \%$ CI $73,709-93,945)$ reported not having ceftriaxone. Access to both of these drugs was generally higher in the South (Table). Physicians in offices not designated PCMHs were more likely than those in offices designated PCMHs to report lacking on-site availability of ceftriaxone 